\title{
LASER ACCELERATION IN A STRIPED MIRROR WAVEGUIDE
}

\author{
A.V. Smirnov, D. Yu, DULY Research Inc., Rancho Palos Verdes, CA 90275
}

\section{Abstract}

Fresnel diffraction dominated fields propagating at glancing angles in a wide planar and rectangular optical waveguides are considered. Both schemes are characterized numerically with multi-harmonic interaction. Resonant effect in terms of the energy gain versus geometry parameter is demonstrated. It is found that adjacent harmonics play a dominant role in the performance at short wavelengths. Performance parameters are calculated for proof-of-principle experiment with moderate laser beam intensities.

\section{INTRODUCTION}

An idea of resonant short-wavelength acceleration of relativistic charged particles in a vacuum periodic structure was proposed many years ago [1]. It was based, in fact, on interaction of a thin straightforward charged particle beam with synchronous harmonics of the field propagating in an oversized, closed [2] or open periodic structure [3]. Single-wave interaction was considered in [3] analytically as a proof of the scheme feasibility. In particular, the synchronism condition was derived for periodic structure as equality between twice the Fresnel number $2 \mathrm{~N}_{\mathrm{f}}$ and the number $\mathrm{q}$ corresponding to the resonant (synchronous) spatial harmonic. For a real multicell structure of finite length the number of spatial harmonics is defined by the number of cells $\mathrm{N}_{\mathrm{c}}$. Therefore, the condition of periodicity in terms of synchronism can be easily reformulated as $\mathrm{N}_{\mathrm{c}}>2 \mathrm{~N}_{\mathrm{f}}$. The same relationship was formulated, in fact, by S. Kheifits [4] for conventional circular structures.

As the wavelength decreases, a growing number of spatial harmonics approach synchronism and interact with the beam. Main performance parameters were calculated numerically for a real structure of finite length for two variants.

\section{THE SIMPLEST DESIGNS}

The principal schemes of plain mirror waveguide are shown in Fig. 1. The first variant is a wide planar structure with linearly polarized laser beam (lowest order Gauissian beam). The second variant is an axially symmetric rectangular (quadratic) structure with two laser beams of different polarizations (with $90^{\circ}$ shift). To characterize numerically the fields in periodic, open structures we consider here the diffracted fields as is, i.e. without artificial extraction of the synchronous Fourier harmonic. The model applied is similar to [3], and is based on Vainshtein's theory [5] along with the method of transition from single open cavity to a periodic open waveguide. Unlike the previous model it does not use the assumption of an infinitely long (periodic) structure.

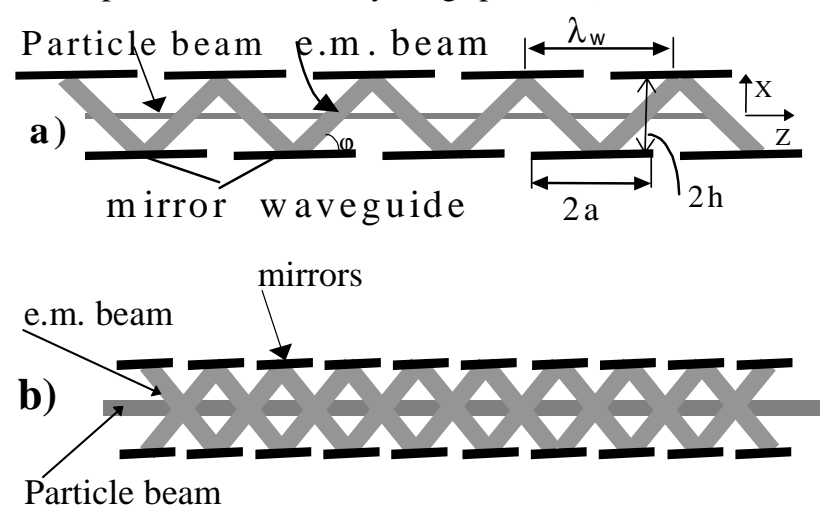

a)

b)
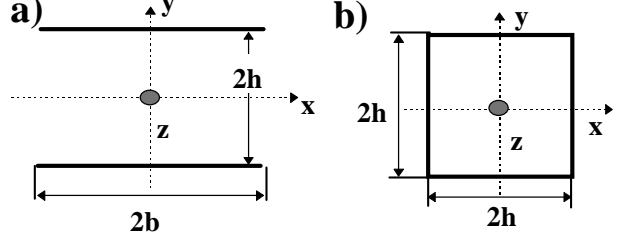

Figure 1: Schematic view of a wide planar striped mirror structure (a) and a rectangular structure (b).

\subsection{Planar Design}

In the calculations presented in this paper we assume a relatively moderate incident laser field intensity: $\mathrm{I}=10^{15} \mathrm{~W} / \mathrm{m}^{2}$, which is below the maximum levels used in a proposal based on lens waveguide [6] using the $\mathrm{BNL} \mathrm{CO}_{2}$ laser accelerator test facility [7]. Correspondingly, we assume a beam energy of $38 \mathrm{MeV}$, a geometry aspect ratio $\mathrm{b} / \mathrm{h}=3$, and a basic acceleration length $\mathrm{L}=11.5 \mathrm{~cm}$.

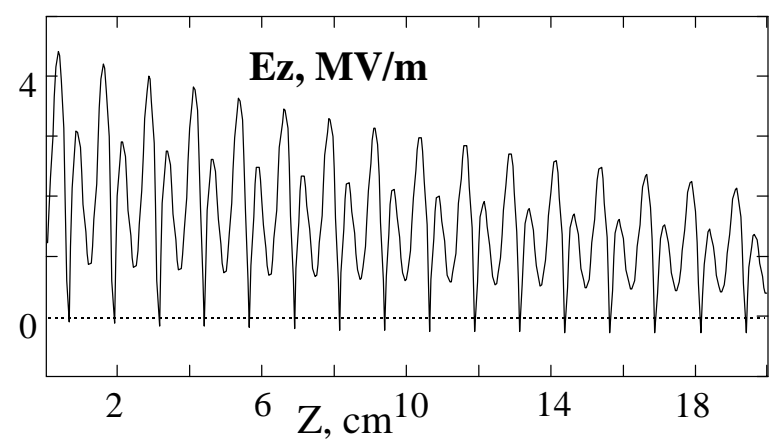

Figure 2: Longitudinal field seen by particle along the wide planar structure.

For the simplest planar design based on single laser, beam we assume the structure period $\lambda_{\mathrm{w}}=2.5 \mathrm{~cm}$.

Using an iterative procedure we found optimal values for injection angle $\varphi=0.038 \mathrm{rad}$, vertical shift of the beam alignment $\Delta \mathrm{y}=50 \mu \mathrm{m}$, aperture gap $2 \mathrm{~h}=0.474 \mathrm{~mm}$ and 
injection phase $\psi=0.6 \mathrm{rad}$. It gives an attenuation length $\mathrm{L}_{\text {att }}=12.3 \mathrm{~cm}$, resonant harmonic number $\mathrm{q}=2$, and $\mathrm{a}$ minimal (longitudinal) Fresnel number $\mathrm{N}_{\mathrm{f}}=1.5$.

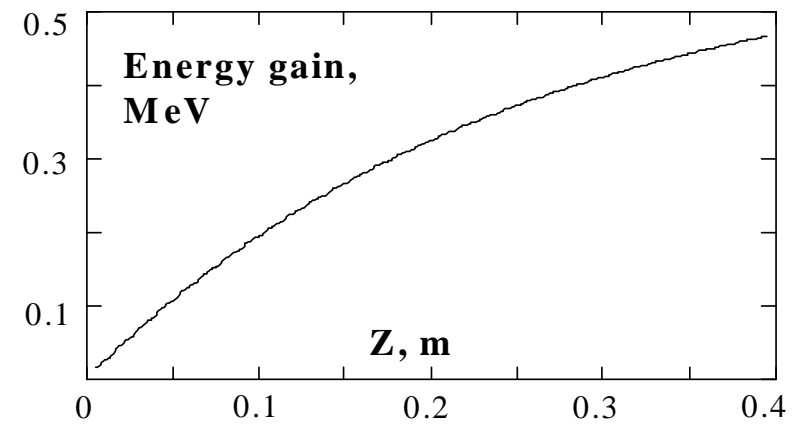

Figure 3: Energy gain along the wide planar structure

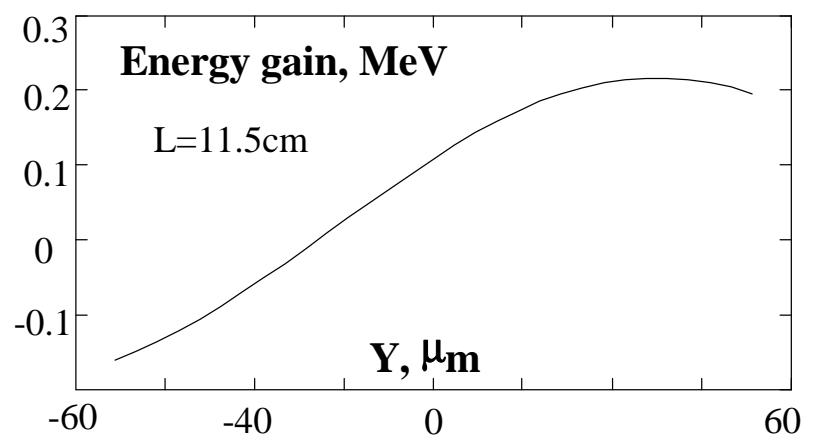

Figure 4: Energy gain vs vertical beam alignment

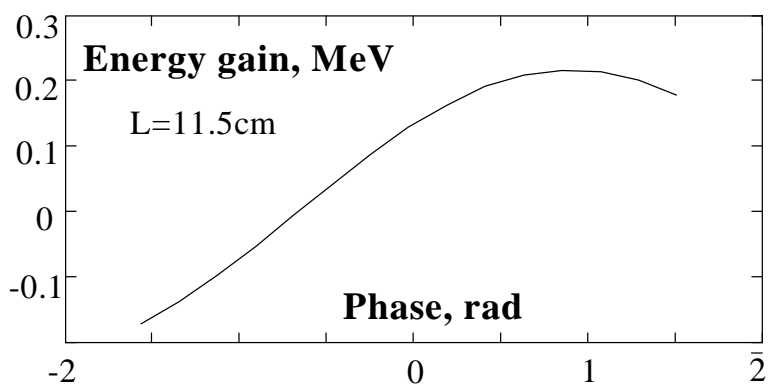

Figure 5: Energy gain vs injection phase

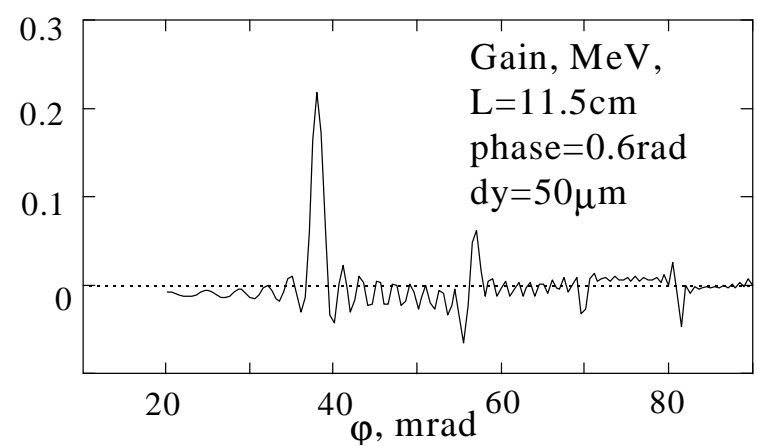

Figure 6: Energy gain as a function of laser beam injection angle (variable consistently with the gap).

The results for the field calculations are illustrated in Figs. 2-6. As it is seen from Fig. 2, the acceleration gradient is negative at some points due to the effect of competition between harmonics close to synchronism. Note, the synchronous harmonic is even $(\mathrm{q}=2)$ and this harmonic itself does not contribute to energy gain [3]. It means that this mode of operation possesses a finite length of synchronism, which usually exceeds the attenuation length. Since we use the lowest laser mode, the optimal vertical position of the beam in general is shifted from the axis of the geometry. From Fig. 4 one can define the maximum beam diameter of $\sim 10-15 \mu \mathrm{m}$. Fig. 6 illustrates the resonant character of the scheme performance with respect to geometry variation (vertical gap $2 \mathrm{~h}$ ), consistent with the laser beam injection angle $\varphi$ : $h(\varphi)=\tan (\varphi) \lambda_{w} / 4$. It is interesting to note that the peaks in the Fig. 6 correspond approximately to the synchronous harmonic numbers $2,4,6,8, \ldots$... From Fig.6 one can estimate the tolerance to the injection angle to be about $0.05-0.1 \mathrm{mrad}$. For higher Fresnel and harmonic numbers the tolerances are proportionally more stringent.

Thus, the practical design of the structure is always a compromise between the finite spread of the injection angle and long enough acceleration length defined by a diffraction figure-of-merit (Fresnel number).

\subsection{Rectangular Design}

To improve performance at the same peak energy fluency on the surface, one can use a rectangular waveguide with two synchronized laser beams of lowest mode injected symmetrically in both horizontal and vertical planes (see Fig. 1b). Unlike 'donut-shaped' laser mode, the resulting field is not radially polarized.

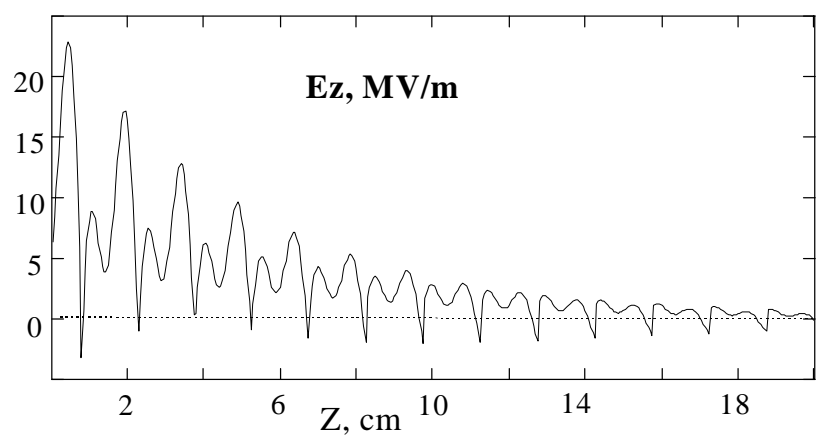

Figure 7: Longitudinal field produced by four laser beam seen by particle along the rectangular structure.

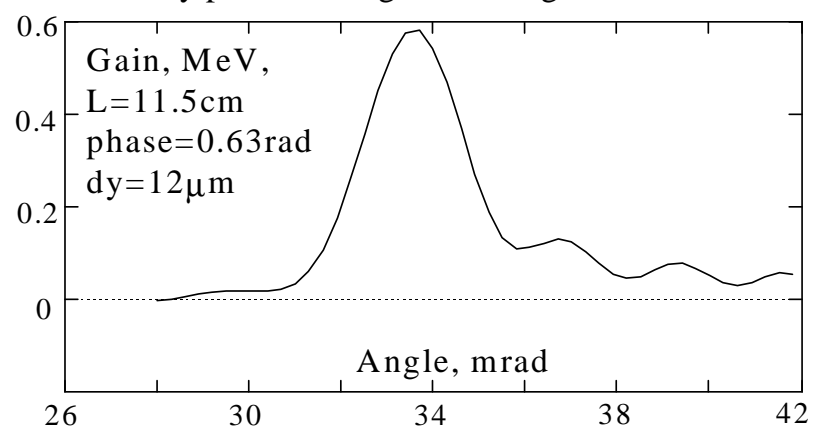

Figure 8: Energy gain as a function of laser beam injection angle (variable consistent with the gap).

In these calculations we assume an incident laser field intensity $\mathrm{I}=10^{15} \mathrm{~W} / \mathrm{m}^{2}$ for each of the laser beams and a structure period $\lambda_{\mathrm{w}}=3 \mathrm{~cm}$ for the symmetric rectangular 
design. Using an optimizing numerical procedure we defined the injection angle $\varphi=0.0336 \mathrm{rad}$, vertical shift of the beam alignment $\Delta \mathrm{y}=12 \mu \mathrm{m}$, aperture gap $2 \mathrm{~h}=1.008 \mathrm{~mm}$ and injection phase $\psi=0.63 \mathrm{rad}$. It gives an attenuation length $\mathrm{L}_{\text {att }}=1.4 \mathrm{~cm}$, an effective resonant number $\mathrm{q}=2.2$, and a minimal (longitudinal) Fresnel number $\mathrm{N}_{\mathrm{f}}=0.157$.

Thus usage of four laser beams results in only a factor of $\sim 3$ increase of energy gain (at given length), basically because of higher diffraction attenuation. Since the area of the mirrors composing the waveguide is considerably smaller compared to previous variant, the radiation loss is increased dramatically (see Fig. 7). On the other hand, sensitivity to the injection angle misalignment is decreased by one order (see Fig. 8). The last circumstance makes the scheme more feasible for proof-of-principle experiment.

\section{DISCUSSION}

The scheme proposed provides a larger gap for the beam transport compared to lens waveguide, and less stringent requirement on the damage fluency due to usage of metal material at glancing angles (versus dielectric at normal angles, see e.g. [8]). It does not require laser beam conversion into donut-shaped mode. Rectangular (quadratic) waveguide provides acceleration efficiency comparable with that in periodic array of focusing lenses [2]. However, high diffraction loss limits the advantages of resonant multi-harmonic mode of acceleration over longer distances. In Fig. 9 we present two corresponding modifications with enhanced figure-of-merit.
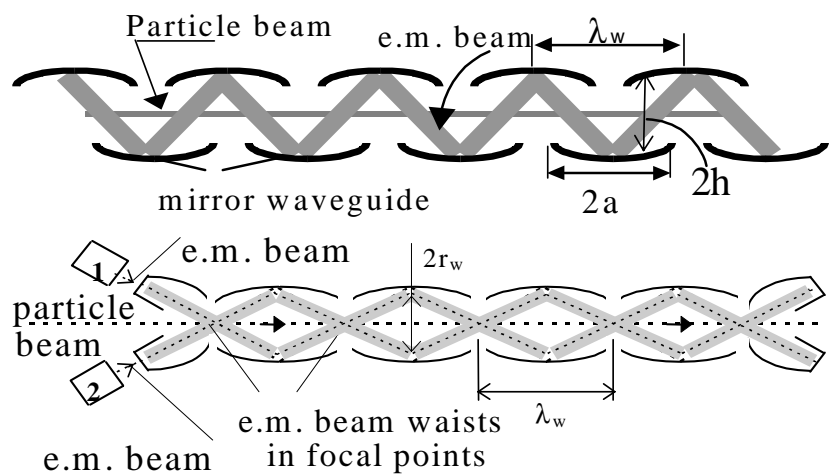

Figure 9: Open mirror waveguides with reduced losses.

For a non-resonant scheme such as OILS [9] one can anticipate an increase of slippage length due to artificial introduction of spatial harmonics close to synchronism. It can be done, e.g., with periodic modulation as shown in Fig. 10. We have found that adjacent harmonics play a dominant role in the performance at short wavelengths in oversized structures. In our case, with only a few periods the harmonics form a quasi-continuous spectrum. This multi-harmonic interaction causes even deceleration at some local points (see Figs. 2,7). A similar situation takes place in the Alternative Gradient scheme [10]. (Also, multi-harmonic interaction was used in ion linac, e.g. in
RFQ invented by Teplyakov in 1964.) Non-synchronous harmonics can have both accelerating and focusing effect in ion and SW electron linacs. Polyharmonic acceleration and focusing of ion beams was studied in $\mathrm{rf}$ ion linac integarated with the electrostatic undulator that can operate even in the absence of synchronous harmonic [11].

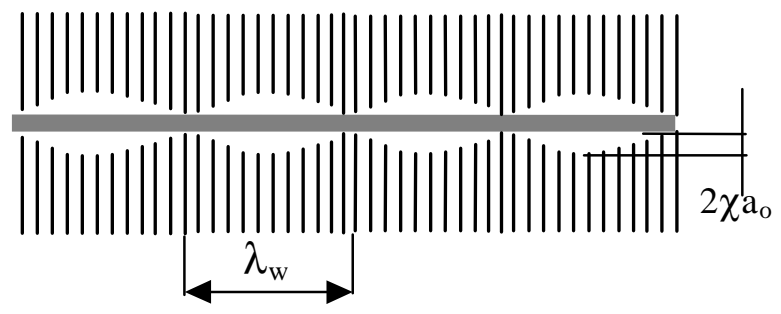

Figure 10: Iris-loaded diffraction-dominated structure with periodically modulated geometry.

\section{ACKNOWLEDGMENTS}

The authors are very appreciate helpful discussions with M. Xie, R. Pantell, D. Whittum, I. Pogorelsky, E.S. Masunov, A.C. Melissinos and R. Tichoplav.

\section{REFERENCES}

[1] A.N. Lebedev, private communications.

[2] A.V. Smirnov, in Proc. of the 6th European Particle Acceleration Conf., Stockholm, 22-26 June, 1998, p. 824.

[3] A.V. Smirnov, in Proc. of PAC'99 Conf., NY, 1999 March 29th - April 2nd (1999) p. 3615.

[4] S. A. Heifets, S. A. Kheifets, SLAC-PUB-4625 (1988).

[5] L.A. Vainshtein, Open resonators and waveguides, 'Sov. Radio,' Moscow (1966).

[6] C. Huang, 'Proposed Physics Experiments for Structure-based Laser-Driven Acceleration in a Vacuum,' submitted to BNL for ATF, Hsinchu, Taiwan, May, 2000

[7] I.V. Pogorelsky, I. Ben-Zvi, J. Skaritka, Z. Segalov, M. Babzien, K. Kushe, I.K. Meskovsky, V.A. Lekometsev, A.A. Dublov, Yu. A. Boloshin, G.A. Baranov, in AIP Conf. Proc., 398 (1997) p. 937.

[8] Y.C. Huang, R.L. Byer, in AIP Conf. Proc., 398, (1997) p. 538.

[9] M. Xie, in Proc. of the PAC'97 Conf., Vancouver, 1216 May, 1997, p. 660

[10] M. Xie, in Proc. of EPAC 2000, Vienna, Austria, p. 895.

[11] E.S. Masunov, N.E. Vinogradov, in Proc. of EPAC'98 Conf, Sweden, Stockholm, 22-26 June, 1998, p. 740. 